\section{Erhöhtes Risiko für venöse Thromboembolien bei rheumatoider Arthritis}

Molander $\mathrm{V}$ et al. Risk of venous thromboembolism in rheumatoid arthritis, and its association with disease activity: a nationwide cohort study from Sweden. Annals of the Rheumatic Diseases 2021; 80: 169-175. doi: 10.1136/annrheumdis-2020-218419

Patienten mit rheumatoider Arthritis (RA) haben im Durchschnitt ein erhöhtes Risiko für venöse Thromboembolien (VTE). Dies zeigten bereits mehrere vorherige Studien. Unbekannt war bislang, ob und wieweit die Risikoerhöhung mit der Krankheitsaktivität der RA zusammenhängt. Eine landesweite Kohortenstudie aus Schweden klärt auf.
VTE einschließlich tiefer Venenthrombosen und Lungenembolien sind mit einer erheblichen Morbidität und Mortalität verbunden. Innerhalb einer Studie mit Daten von mehr als 46000 Patienten beurteilten nun schwedische Wissenschaftler die Inzidenz von VTE bei RA im Vergleich zu Personen ohne RA. Sie untersuchten außerdem den Zusammenhang zwischen Aspekten der klinischen Krankheitsaktivität bei RA und dem Risiko einer VTE.

Die landesweite registerbasierte Kohortenstudie wurde zwischen 2006 und 2008 durchgeführt. Die Forscherinnen und Forscher nutzten das schwedische Rheumatologie-Qualitätsregister und identifizierten alle Patienten mit RA mit mindestens einem registrierten Rheumatologenbesuch während des Studienzeitraums ( $n=46316 \mathrm{~Pa}$ tienten, 322601 Besuche). Es wurden durch die Verknüpfung zu anderen nationalen Patientenregistern etwa $90 \%$ aller schwedischen, von Rheumatologen behandelter Patienten mit RA in die Analysen einbezogen. Die Patienten waren durchschnittlich 63 Jahre alt, $74 \%$ von ihnen waren weiblich.

Die Disease Activity Score 28 ErythrozytenSedimentationsrate (DAS28 ESR) und ihre Komponenten dienten als Messgröße. So wurde jeder Besuch nach der erfassten DAS28-Kategorie folgendermaßen hinsichtlich Krankheitsaktivität eingeteilt: Remission (0-2,6), geringe $(2,7-3,2)$, mittlere (3,3$5,0)$ und hohe $(>5,0)$ Krankheitsaktivität. Hauptergebnis war ein VTE-Ereignis innerhalb des auf den Besuch folgenden Jahres. Die Forscherinnen und Forscher schlossen Referenzpersonen aus der Allgemeinbevölkerung, gematcht hinsichtlich Alter, Geschlecht und Wohngegend, im Verhältnis 1:5 ein.

\section{Ergebnisse}

- Basierend auf 2241 inzidenten VTE-Ereignissen innerhalb eines Jahres nach jedem einbezogenen Besuch und 5301 VTE-Ereignissen in der allgemeinen Bevölkerungskohorte betrug das Risikoverhältnis für VTE bei RA 1,88 (95\%-KI 1,65-2,15).

- Bei Patienten mit RA stieg das Risiko (und das Risikoverhältnis) mit zunehmender RA-Krankheitsaktivität von $0,52 \%$ nach Besuchen in Remission auf 1,08\% nach Besuchen mit DAS28 ESR hoher Krankheitsaktivität (Relatives Risiko im Vergleich zur Remission = 2,03, 95\%-KI 1,73-2,38).

- Auch Patienten mit RA in DAS28 ESR-Remission hatten im Vergleich zur Allgemeinbevölkerung ein erhöhtes VTE-Risiko.

\section{FAZIT}

Laut Autorinnen und Autoren gibt es einen starken Zusammenhang zwischen der klinischen RA-Krankheitsaktivität und dem Risiko einer VTE. Die RA-Krankheitsaktivität könne als zusätzliches Instrument zur VTE-Risikostratifizierung bei Patienten mit RA verwendet werden. Von einer direkten Kausalität zwischen den einzelnen Aspekten der RA-Krankheitsaktivität und dem VTE-Risiko könne jedoch anhand der Ergebnisse nicht gesprochen werden.

\section{Annkatrin Wagner, Stuttgart}

